\title{
BIM FROM LASER SCANS... NOT JUST FOR BUILDINGS: NURBS-BASED PARAMETRIC MODELING OF A MEDIEVAL BRIDGE
}

\author{
L. Barazzetti*, F. Banfi, R. Brumana, M. Previtali, F. Roncoroni
}

ABC Department, Politecnico di Milano, Piazza Leonardo da Vinci 32, Milan, Italy (luigi.barazzetti, fabrizio.banfi, raffaella.brumana, mattia.previtali, fabio.roncoroni)@ polimi.it http://www.gicarus.polimi.it

KEY WORDS: BIM, BIMonitoring, Interoperability, Laser Scanning, Photogrammetry, Monitoring, NURBS

\begin{abstract}
:
Building Information Modelling is not limited to buildings. BIM technology includes civil infrastructures such as roads, dams, bridges, communications networks, water and wastewater networks and tunnels. This paper describes a novel methodology for the generation of a detailed BIM of a complex medieval bridge. The use of laser scans and images coupled with the development of algorithms able to handle irregular shapes allowed the creation of advanced parametric objects, which were assembled to obtain an accurate BIM. The lack of existing object libraries required the development of specific families for the different structural elements of the bridge. Finally, some applications aimed at assessing the stability and safety of the bridge are illustrated and discussed. The BIM of the bridge can incorporate this information towards a new "BIMonitoring" concept.
\end{abstract}

to preserve the geometric complexity provided by point clouds, obtaining a detailed BIM with object relationships and attributes.

\section{INTRODUCTION}

Building Information Modelling is becoming a very popular technology for several infrastructures such as utility systems, roads and rails, bridges, dams, tunnels, communications networks, water and wastewater networks, etc. This fosters the use of advanced digital models (including semantics, object relationships and attributes) instead of traditional construction projects still based on 2D and 2D CAD drawings. However, BIM technology has limitations, especially for complex constructions with and irregular constructive elements. This is a significant problem for both modern and existing constructions (Agapiou et al., 2015, Patraucean et al., 2015; Tang et al., 2010; Volk et al., 2014, Cuca et al., 2015)
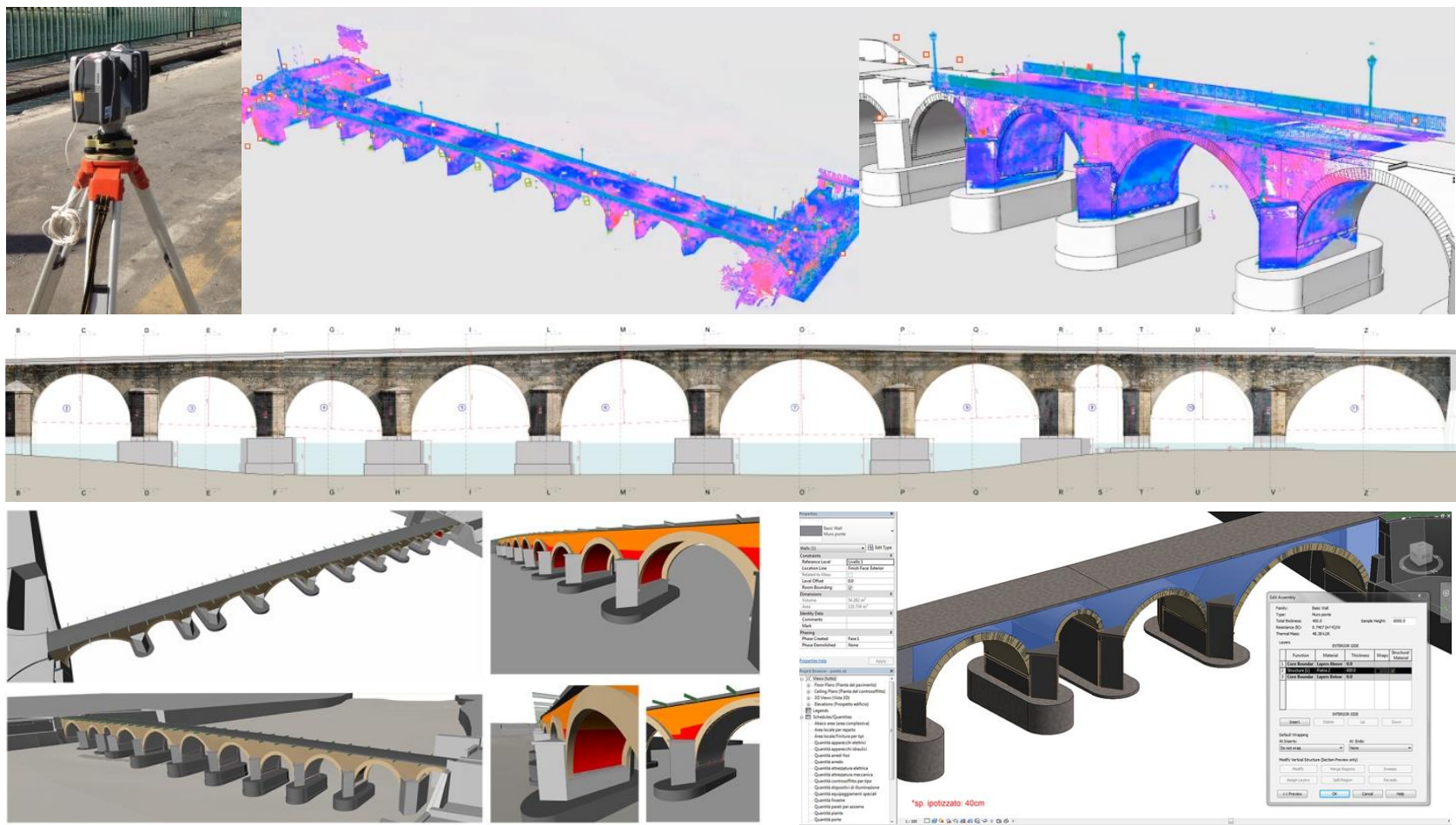

Figure 1. The BIM of the medieval bridge Azzone Visconte (Lecco, Italy) generated from laser scans and digital images coupled with new algorithms and procedures for parametric modelling. This allowed one to preserve the geometric complexity provided by point clouds, obtaining a detailed BIM with object relationships and attributes. 
This paper focuses on BIM for bridges (sometimes referred to BrIM - Bridge Information Modelling), which is a novel approach able to manage the whole lifecycle of a bridge: fabrication, construction, operation, maintenance and inspection. The case study presented in this work is the medieval bridge Azzone Visconte (also known as the Old Bridge) over the Adda River, in Lecco (Italy). The bridge was built between 1336 and 1338 and today is the symbol of the city (Fig. 2). Several analyses were carried out to assess the stability and safety of the bridge, as well as the state of conservation of materials and structures.

A geometrical survey at the scale 1:50 was one of the required products of the project. The complex and irregular geometry of the bridge resulted in several limitations when the modelling tools in BIM software packages were used. It should be mentioned that this "geometric" problem is not limited to historic objects. It also includes new bridges often characterized by variable curvature and cross sections. From this point of view, the lack of powerful BIM instruments able to reconstruct complex shapes is still a major drawback in BIM projects, and the generation of accurate historic BIM (Murphy et al., 2013) surveyed from laser scanning point clouds is a challenging task which requires the development of new libraries or the implementation of new algorithms (some examples are discussed in Fai et al., 2011; Baik et al., 2015; Oreni et al., 2014; Dore et al., 2015; Quattrini et al., 2015, Barazzetti et al., 2015c).

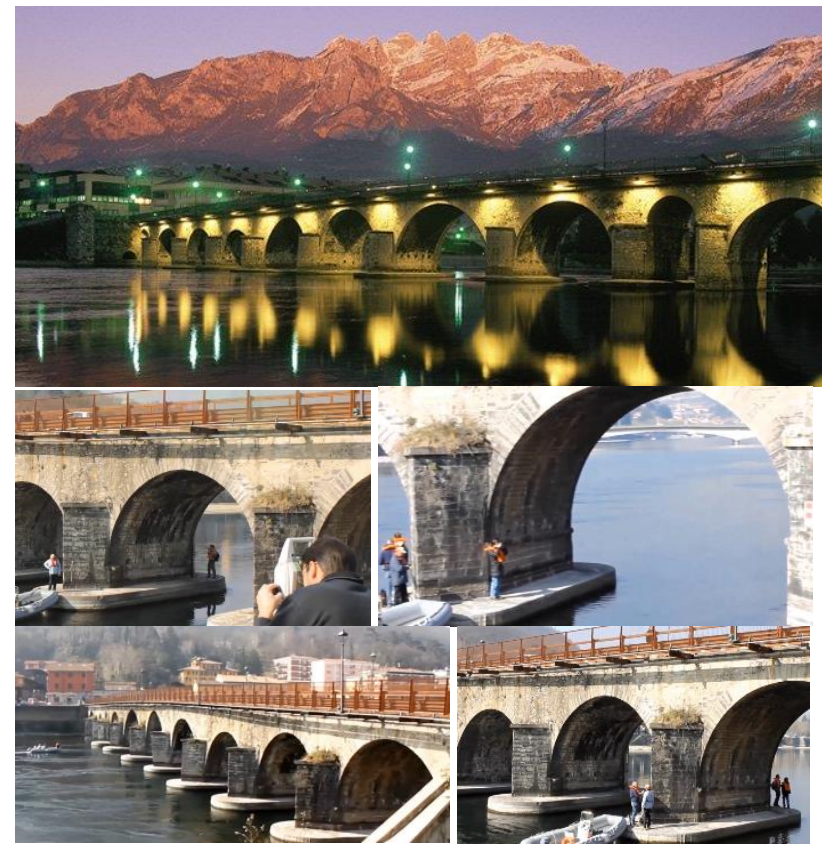

Figure 2. The Old Bridge of Lecco (Italy) and some pictures of the data acquisition phase.

In addition, the use of software for "pure" (direct) 3D modelling was not possible (e.g. Rhinoceros, AutoCAD, Maya, 3D Studio Max). Such software can provide 3D models without parametric modelling tools, object relationships and attributes (Eastman et al., 2008).

BIM requires a database including semantics and object properties to create and manage meaningful information about the construction. BIM software packages (e.g. Revit, ArchiCAD, AECOsim Building Designer, Teckla, etc.) allow users to electronically collaborate at different levels with a consistent exchange of digital information.

This paper describes a procedure for BIM generation able to take into consideration the geometric complexity captured by laser * corresponding author scanning point clouds. The implemented solution for as-built BIM generation (from laser point clouds and photogrammetry) is based on NURBS curves and surfaces (Piegl and Tiller, 1997) converted into BIM objects. The final BIM objects are then imported in the commercial package Autodesk Revit to ensure a consistent exchange of information among the different specialists involved in the project.

\section{SCAN ACQUISITION AND REGISTRATION}

The geometric survey of the bridge was carried out with laser scanning and photogrammetric techniques. Data were registered in a stable reference system given by a geodetic network (Fig. 3) measured with a Leica TS30. The network is made up of 6 stations and the measurement phase took one day. In all, 834 observations and 264 unknowns gave 570 degrees of freedom. Least Squares adjustment provided an average point precision of about $\pm 1.5 \mathrm{~mm}$.

The complexity and the size of the bridge required 77 scans registered with the geodetic network. The instrument is a Faro Focus 3D and the final point cloud is made up of 2.5 billion points. The instrument was placed in different positions, including the road and the riverbanks. The survey of the vaults required the creation of a mobile metal structure that allowed one to capture the intrados (Fig. 4).

The network provided a robust reference system to remove deformations during scan registration. Scans were registered with an average precision of $\pm 3 \mathrm{~mm}$ by using chessboard targets measured with the total station and additional scan-to-scan correspondence (spherical targets).

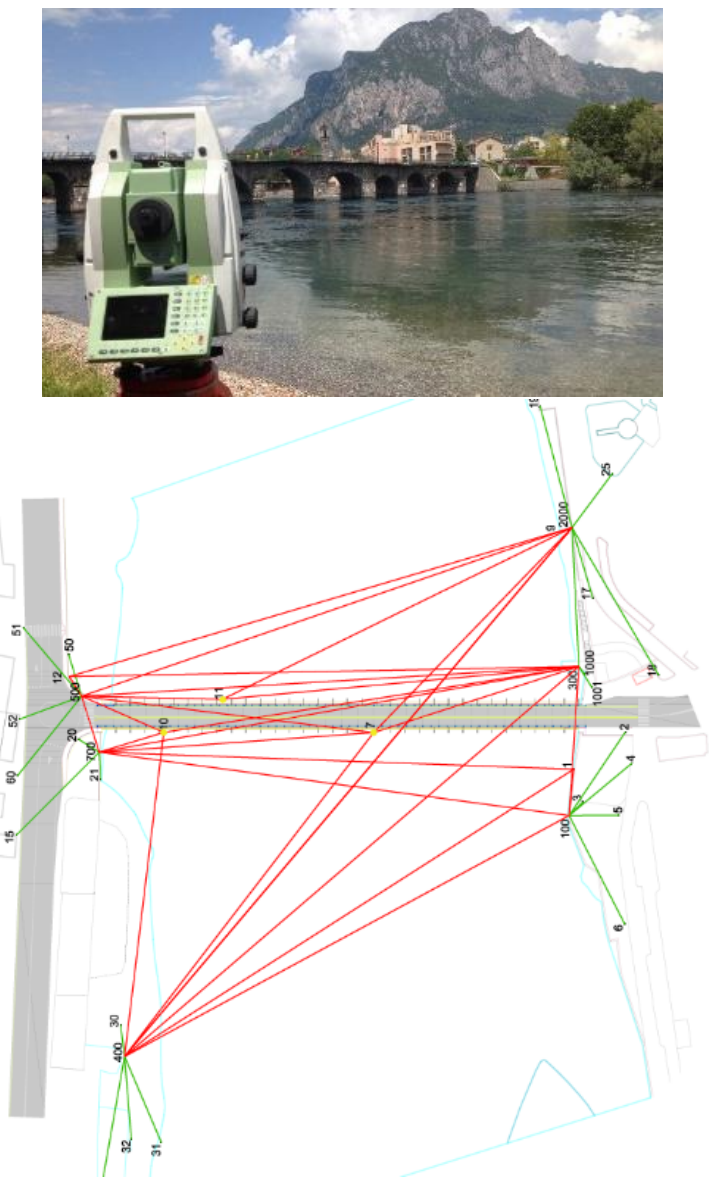

Figure 3. The geodetic network measured with a total station Leica TS30 (average point precision is $\pm 1.5 \mathrm{~mm}$ ). 
The laser scanning survey was then integrated with more than 500 images captured from a boat. Photogrammetry was used to generate accurate orthophotos of the fronts (South and North), the columns and the vaulted surfaces (intrados). The use of a total station data allowed one to obtain a common reference system for the different acquisition techniques.

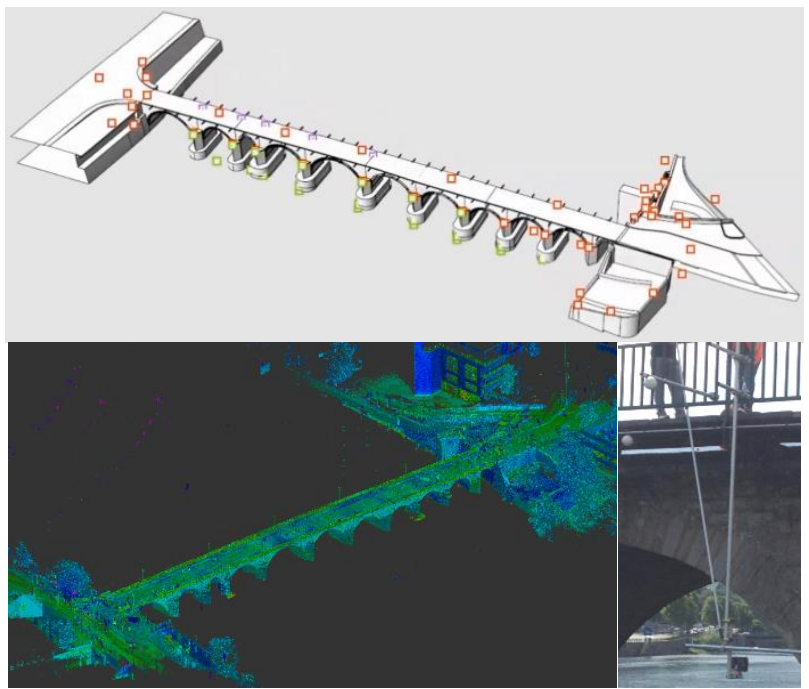

Figure 4. Scan positions (top), a 3D view of the registered point clouds (bottom left), and the metal structure used to lower the laser scanner (bottom right).

As the goal of the project is a BIM useful to assess the stability of the bridge, the surveying phase cannot be limited to the reconstruction of the shape. The presented measurement techniques can reveal the external layer of construction elements, whereas a BIM is made up of objects with an internal structure. As the goal is the creation of an interoperable BIM for different specialists (engineers, architects, historians, archaeologists, restorers, etc.), the survey included a historical analysis, the identification of materials, technological aspects, stratigraphic analysis, and information from other inspections such as destructive inspections and IR thermography.

\section{GENERATION OF THE BIM}

The starting point for the generation of the BIM is the set of dense laser scanning point clouds which reveal the geometric complexity of the bridge. Photogrammetry and laser scanning are useful technologies for irregular surfaces. On the other hand, BIM projects require an object-based representation made up of solid elements with relationships and attributes. The complexity of the bridge, with irregular shapes not available in existing object libraries, required the development of procedures for parametric modelling able to overcome the lack of commercial software able to preserve the level of detail encapsulated into laser scanning point clouds.

For this reason, a procedure based on surfaces made up of NURBS curves and NURBS surfaces (Piegl and Tiller, 1997) was used to create parametric BIM objects.

\subsection{Creation of 3D curves}

NURBS are mathematic functions used in CAD projects to model simple shapes and complex free-forms objects. Although NURBS are very advanced mathematical objects, they can be used for manual modelling with an easy-to-understand geometric interpretation. Several commercial packages are based on
NURBS (e.g. Ashlar-Vellum CAD, Blender, EvoluteTools PRO, Hexagon, Maya, Mol, Nurbana 3D, Rhinoceros, ...). However, the result achievable with these software is only a 3D model, which is not a BIM for the lack of basic parametric modelling requirements.

The proposed procedure for surface reconstruction uses a preliminary extraction of NURBS curves (in space) from the point cloud. Such curves are not random curves in space. They follow the logic of construction of the bridge, which is therefore divided into its constructive elements.

A point cloud provides the geometric information needed to estimate the parameters of NURBS curves. A subset of the original 3D points can be used as control points $\left\{\mathbf{P}_{i}\right\}$ for NURBS generation. Given a set of $n+1$ control points $\mathbf{P}_{0}, \ldots, \mathbf{P}_{n}$ a NURBS curve of degree $p$ is defined by:

$$
\mathbf{C}(u)=\frac{\sum_{i=0}^{n} N_{i, p}(u) w_{i} \mathbf{P}_{i}}{\sum_{i=0}^{n} N_{i, p}(u) w_{i}}
$$

where $\left\{w_{i}\right\}$ are the weights and the $\left\{N_{i, p}(u)\right\}$ are the $p$ th-degree $\mathrm{B}$-spline basis functions defined on the knot vector $U$, which is a non-decreasing sequence of real numbers whose elements are called knots.

The $i$ th B-spline basis function $N_{i, p}(u)$ has the recursive CoxdeBoor form:

$$
\begin{gathered}
N_{i, 0}(u)=\left\{\begin{aligned}
1 & \text { if } u_{i} \leq u \leq u_{i+1} \\
0 & \text { otherwise }
\end{aligned}\right. \\
N_{i, p}(u)=\frac{u-u_{i}}{u_{i+p}-u_{i}} N_{i, p-1}(u)+\frac{u_{i+p+1}-u}{u_{i+p+1}-u_{i+1}} N_{i+1, p-1}(u)
\end{gathered}
$$

Shown in Fig. 5 are some functions that show the intrinsic high level of manipulation of NURBS curves: (top) a $3^{\text {rd }}$ degree NURBS curve defined by 7 control points $(0, \ldots, 6)$ control points with knot vector $\mathbf{k}_{1}=\{0,0,0,0,1 / 4,1 / 2,3 / 4,1,1,1,1\}$, and (bottom) the variation with a simple modification of the knot vector $\mathbf{k}_{2}=\{0,0,0,0,1 / 4,1 / 4,1 / 4,1,1,1,1\}$. The control points (blue dots) are the same and both knot vectors start (and end) with a knot that has multiplicity $p+1$. Curves with such knot vectors have the remarkable property to start and end in a control point $\mathbf{C}(0)=\mathbf{P}(0), \mathbf{C}(9)=\mathbf{P}(9)$. The first knot vector is uniform because it starts with a full multiplicity knot followed by simple knots equally spaced, and it terminates with a full multiplicity knot.

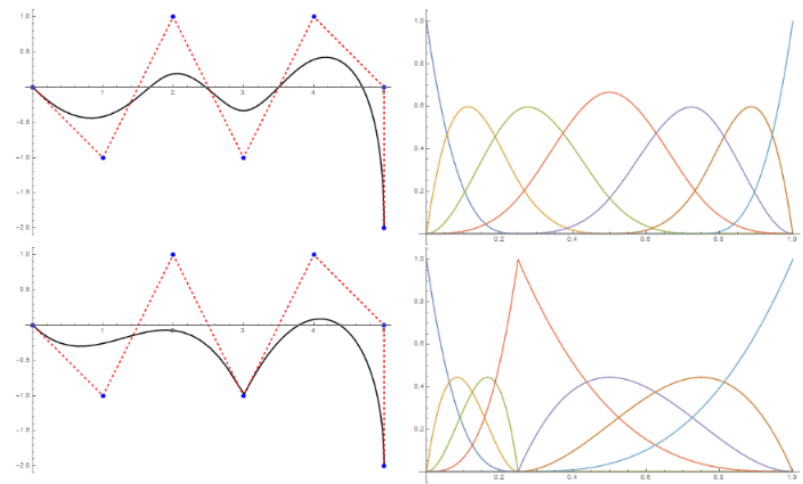

Figure 5. The variation of planar NURBS curves with equal control points (left) and different basis functions (right).

Knots can also be non uniform. For instance, the vector $\mathbf{k}_{2}$ is associated to a change in the basis functions that gives the new curve where $\mathbf{C}(4)=\mathbf{P}(4)$. The modification of the knot vector has 
an influence on continuity (smoothness). In particular, duplicated knots in the middle make a NURBS curve less smooth. The extreme case is a full multiplicity knot in the middle, which corresponds to a point in the curve that becomes a kink.

The degree of the curve is also useful to define the shape of the curve. Shown in Fig. 6 are NURBS curves with common control points and variable degree ( $p$ equals $1,2,3$, respectively). As can be seen, the linear case $(p=1)$ represents the typical polyline (zero-order continuity), whereas a high degree is smoothing the curve. In practical application NURBS with degree up to 5 are usually used.

Since NURBS are rational functions, they can represent freeform entities, but also exact conics such as ellipses, circles and hyperbolas. This confirms their flexibility to design a large variety of shapes, from straight lines and polylines to free-form curves with arbitrary shapes.

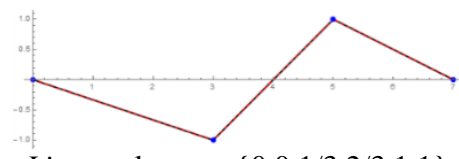

Linear - knots $=\{0,0,1 / 3,2 / 3,1,1\}$
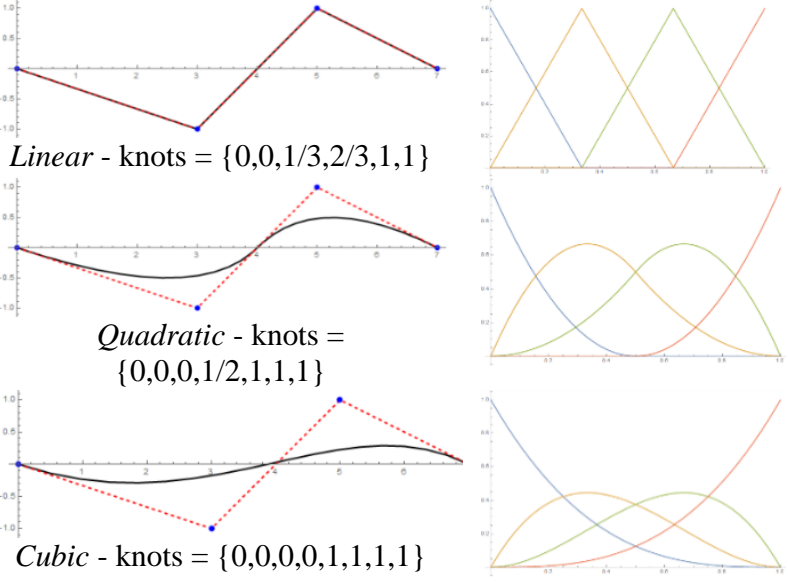

Figure 6. The degree controls the smoothness of the NURBS curves.

Finally, the shape of the curve can be modified by moving its control points. This is the most common way to make local adjustments, especially in interactive modeling projects. Interactive modifications can be carried out by dragging the control points: efficient algorithms provide real-time estimation of unknown parameters and visualization of the final result.
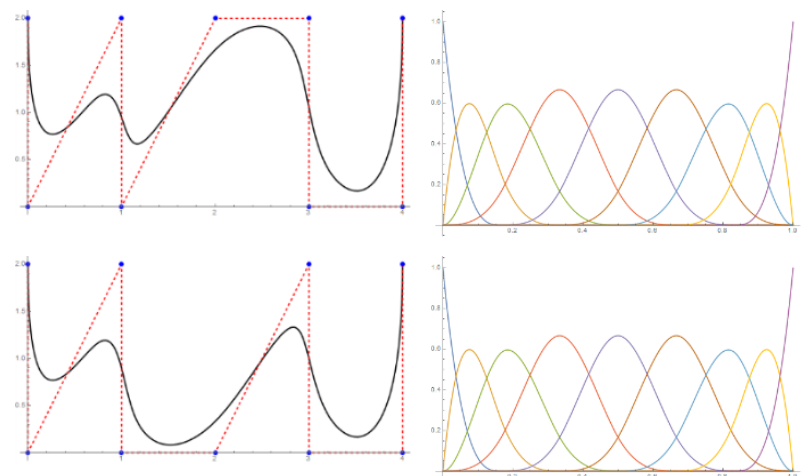

Fig. 7. A NURBS can be locally modified by changing its control points. This does not affect the whole profile of the curve.

As mentioned, one remarkable property consists in the opportunity to generate local variations without affecting the global shape of the curve. Shown in Fig. 7 are two curves (degree 3 with exactly the same (uniform) knot vector $\{0,0,0,0,1 / 6,1 / 3,1 / 2,2 / 3,5 / 6,1,1,1,1\}$ and control points, except for the displacement of a point in the middle. The change in curve's shape is carried out only in a small area near the control point.

The previous figures show that NURBS curves are very efficient tools for modelling simple and complex shapes, small and large objects with variable level of details, as well as regular and irregular parts. The manipulation of knots, control points, weights and degree, allowed one to design the vast range of shapes of the bridge (Fig. 8) and refine the first "visual" result in real time thanks to fast and memory efficient computational algorithms that preserve mathematical exactness.
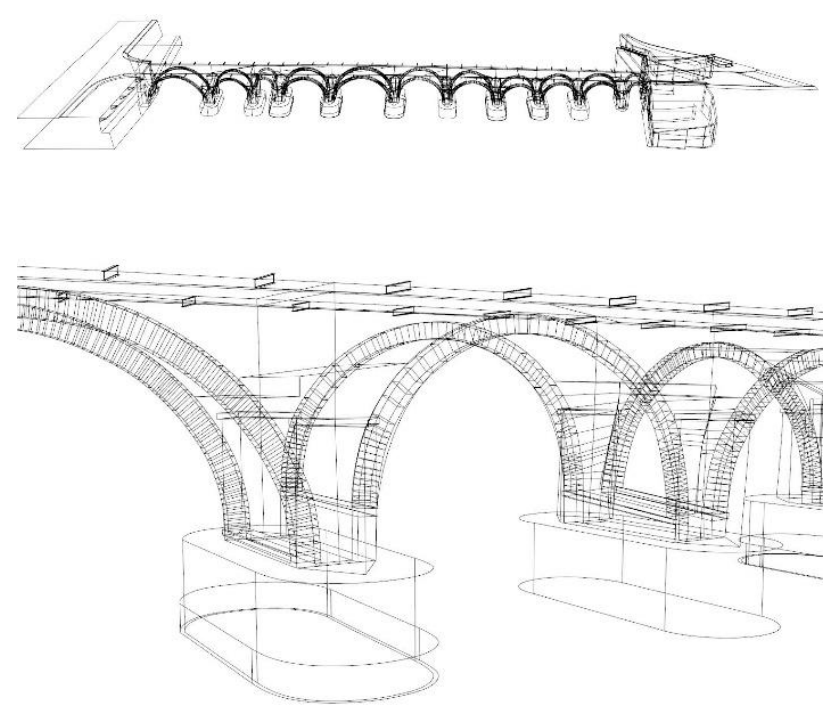

Figure 8. NURBS curves used to drive the generation of BIM objects.

\subsection{Creation of BIM objects from 3D curves}

$3 \mathrm{D}$ curves can be included in surface modeling as additional constraints, so that the congruence of consecutive surfaces is guaranteed by a curve that becomes an edge.

A NURBS surface of degree $(p, q)$ in the directions $(u, v)$ is defined by:

$$
\mathbf{S}(u, v)=\frac{\sum_{i=0}^{n} \sum_{j=0}^{m} N_{i, p}(u) N_{j, q}(v) w_{i, j} \mathbf{P}_{i, j}}{\sum_{i=0}^{n} \sum_{j=0}^{m} N_{i, p}(u) N_{j, q}(v) w_{i, j}}
$$

where $\left\{w_{i, j}\right\}$ are the weights, and the $\left\{N_{i, p}(u)\right\}$ and $\left\{N_{j, q}(v)\right\}$ are the B-spline basis functions defined on the knot vectors $U$ and $V$. NURBS surfaces can be used to represent both complex and predefined mathematical surfaces, such as cylinders, spheres, paraboloids, and toroidal patches. In addition, they can represent ruled surfaces by means of a generatrix (i.e. a $q$ th-degree NURBS curve $\mathbf{C}(v)=\sum_{j=0}^{n} R_{j, q}(v) \mathbf{P}_{j}$ on the knot vector $V$ ) revolved around an axis. Cylinders and cones are typical examples that can be also intended as surfaces of revolution, but the method becomes extremely useful in the case of historical objects where the generatrix can be digitized from the point clouds. This makes NURBS useful for both simple and complex shapes.

In the case of the bridge, the solution for surface generation was based on the use of multiple curves, then NURBS surfaces were generated from one, two or a set of curves in space, which are used as geometric constraint for surface interpolation. Although NURBS surfaces can be fitted to an unorganized point cloud, the final representation of sharp features is usually very poor. For this reason, the use of a preliminary set of curves for the generation of the surface was a better solution. Indeed, the creation of a curve network which drives the surface is much 
easier than a direct manipulation of the surface, especially in the case of discontinuity lines.

After the extraction of the principal discontinuities, which provide the skeleton of a 3D shape, surfaces were generated with an interpolation of the curves through one or more surfaces. The curve should be interpolated as closely as possible so that the distance between the curves, clouds, and final surface is minimum (Hu et al., 2001; Brujic et al., 2002).

The reconstruction of the bridge included the preliminary subdivision of the bridge into structural elements following the logic of construction (how the bridge is actually built, such as foundations, columns, arches, etc., see Brumana et al., 2013). This process allows an accurate geometric representation of the external shape surveyed with laser scanning technology and photogrammetry. On the other hand, it does not provide BIM objects for the lack of parametric modelling tools, relationships between objects and attributes. In addition, point clouds reveal precious metric information about the external first layer of the different elements, i.e. the visible layer that can be surveyed with images and range data, whereas a BIM must incorporate additional information concerning volume (e.g. thickness), material properties, and the organization of structural elements.

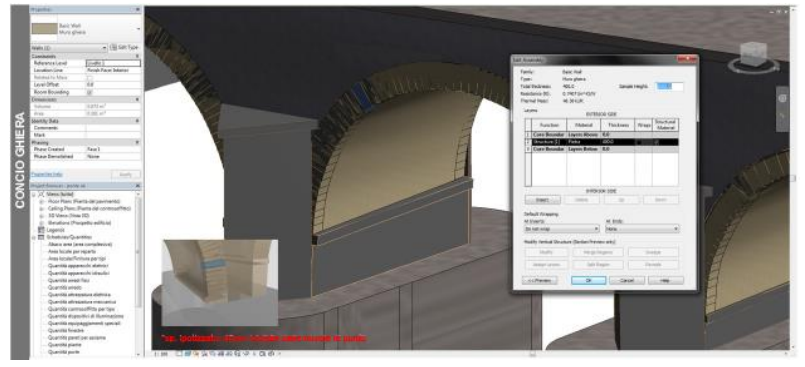

Figure 9. A detail of the BIM in Revit and the opportunity to select objects obtaining specific information.

As NURBS are univocally described by a finite set of parameters (degree, a set of weighted control points, and a knot vector), the main idea for their BIM representation is a mathematical solution that (i) preserves the original parameters and (ii) adds new objectbased information including geometric data and attributes. NURBS surfaces are therefore used to generate the external shape of BIM objects, which are then imported in the commercial software Autodesk Revit. Shown in Fig. 9 are some BIM objects used in the project (ashlars and foundations). Structural elements were classified following the predefined structure of the software database: category, family, type, and instance, obtaining and interoperable model for the different specialists involved in the project.

\section{BIMonitoring: A NOVEL MULTI-USER APPROACH FOR INTEGRATED PROJECTS}

The BIMonitoring approach proposed in this paper starts from a simple consideration: the availability of a BIM (in a multidisciplinary project in which different specialists are involved in the monitoring process, such as engineers, architects, conservators, historians, geologists, geophysicists, etc.) provides a common platform to (i) store results and reports and (ii) to perform new analysis.

In the case of the bridge, different numerical and graphical results were available thanks to the contribution of different specialists: displacements measured during the loading phase to assess the load capacity of the bridge (Fig. 10), GPR (Ground Penetrating Radar) data to inspect the internal structure of the bridge, mapping of cracks, stratigraphy, coring, and mechanical characterization of specimens.
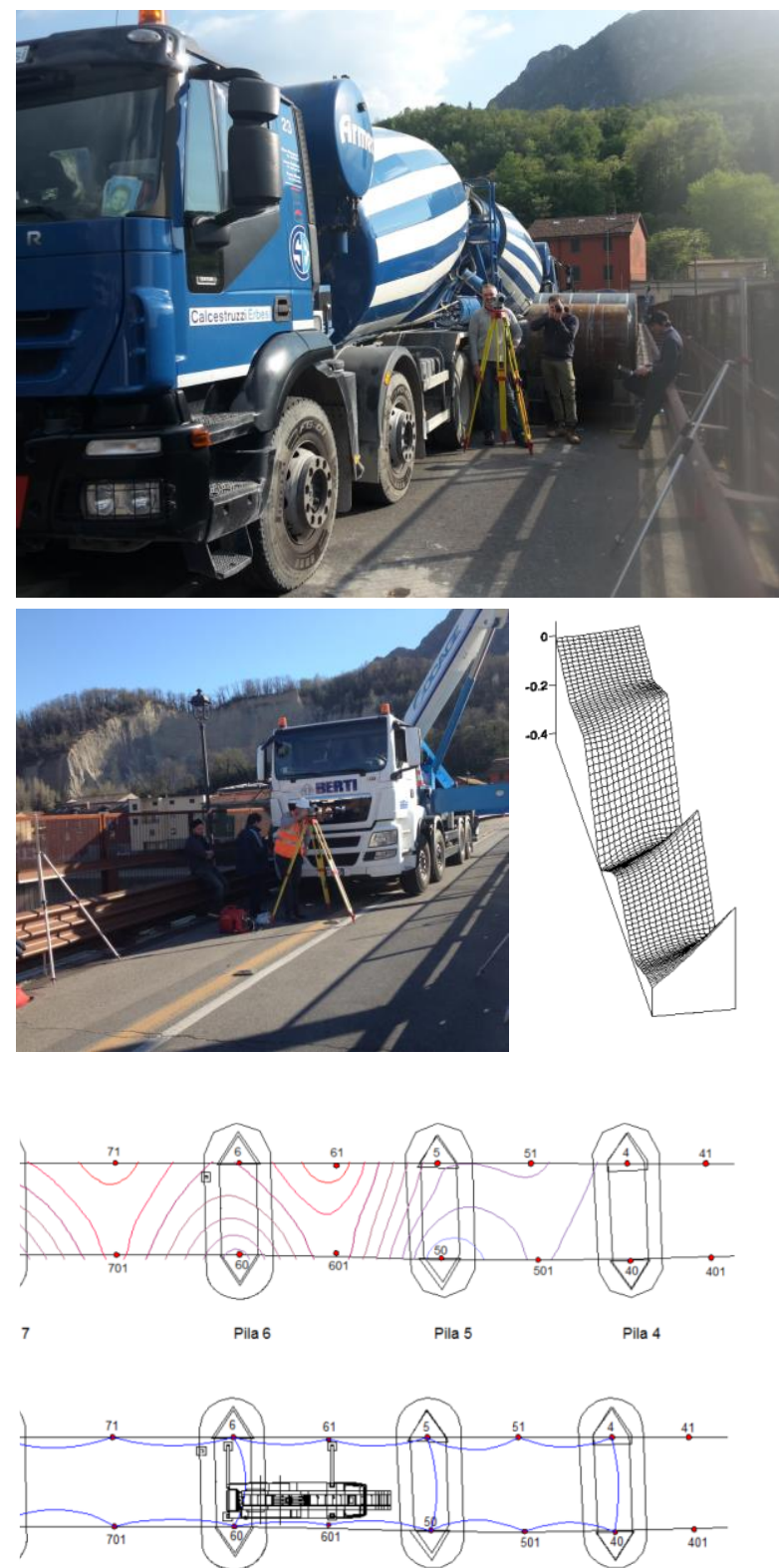

Figure 10. Measurement of vertical displacements by geometric levelling during the loading phase of the bridge.

This kind of information can be directly correlated to 3D information and can be stored in different ways in the BIM, also including links to reports, images, and videos. From this point of view, the use of a $3 \mathrm{D}$ model in a reference system allows the integration of information like in a traditional GIS software used in cartography. The 3D model of the BIM is a graphical representation that allows users to easily interact with the database of the construction.

Moreover, the BIM is not intended only as a database where information is stored. Additional analysis can be performed by using products directly generated from the BIM. An example is the finite element analysis (FEM) to assess structural stability. Previous works (Barazzetti et al., 2015a; 2015b) demonstrated that this approach is feasible. In the case of the bridge, a numerical analysis will be carried out with a simplified geometric model generated from the original BIM. 


\section{CONCLUSIONS}

This paper presented an approach for BIM generation of a medieval bridge, demonstrating that the traditional BIM approach for buildings can be exploited also for complex infrastructures.

The proposed approach tried to overcome some limitations of commercial BIM software packages. Indeed, there is a lack of commercial software able to provide accurate BIM reconstructions of complex geometries, including parametric modelling tools, relationships between objects, and attributes.

The BIM reconstruction of the medieval bridge required the development of new algorithms and procedures. On the other hand, the final result is available in a commercial software so that a single platform becomes available for the different specialists involved in the project.

Finally, a new approach coined BIMonitoring was developed. The BIM can become a shared platform for different category of data in different formats, which can be efficiently integrated towards a sustainable conservation of the structure.

\section{ACKNOWLEDGMENTS}

The work was supported by the project Ponte Azzone Visconti Lecco, Indagini, rilievi e verifiche strutturali.

\section{REFERENCES}

Agapiou, A., Lysandrou, V., Alexakis, D.D., Themistocleous, K., Cuca, B., Argyriou, A., Sarris, A., Hadjimitsis, D.G., 2015. Cultural heritage management and monitoring using remote sensing data and GIS: The case study of Paphos area, Cyprus. Computers, Environment and Urban Systems, 54, pp. 230-239.

Baik, A., Yaagoubi, R., Boehm, J., 2015. Integration of Jeddah Historical BIM and 3D GIS for Documentation and Restoration of Historical Monument. 25th International CIPA Symposium 2015 (XL-5/W7), Taipei, Taiwan, pp. 29-34.

Barazzetti, L., Banfi, F., Brumana, R., Gusmeroli, G., Oreni, D., Previtali, M., Roncoroni, F., Schiantarelli, G., 2015a. BIM from laser clouds and finite element analysis: combining structural analysis and geometric complexity. The International Archives of the Photogrammetry, Remote Sensing and Spatial Information Sciences, Volume XL-5/W4, pp. 345-350.

Barazzetti, L., Banfi, F., Brumana, R., Gusmeroli, G., Previtali, M., Schiantarelli, G., 2015b. Cloud-to-BIM-to-FEM: Structural simulation with accurate historic BIM from laser scans. Simulation Modelling Practice and Theory, Volume 57, September 2015, pp. 71-87.

Barazzetti, L., Banfi, F., Brumana, R., Previtali, M., 2015c. Creation of Parametric BIM Objects from Point Clouds Using NURBS. The Photogrammetric Record, Vol. 30(152), 339-362.

Brujic, D., Ristic, M., Ainsworth, I., 2002. Measurement-based modification of NURBS surfaces. Computer-Aided Design, 24, 173-183.

Brumana, R., Oreni, D., Cuca, B., Binda, L., Condoleo, P., Triggiani, M., 2014. Strategy for integrated surveying techniques finalized to interpretive models in a byzantine church, Mesopotam, Albania. International Journal of Architectural Heritage, 8, pages 886-924.

Cuca, B., Agapiou, A., Kkolos, A., Hadjimitsis, D. 2014. Integration of innovative surveying technologies for purposes of
3D documentation and valorisation of St. Herakleidios Monastery in Cyprus. Lecture Notes in Computer Science (including subseries Lecture Notes in Artificial Intelligence and Lecture Notes in Bioinformatics), 8740, pp. 387-395.

Dore, C., Murphy, M., McCarthy, S., Brechin, F., Casidy, C., Dirix, E., 2015. Structural Simulations and Conservation Analysis -Historic Building Information Model (HBIM). The International Archives of the Photogrammetry, Remote Sensing and Spatial Information Sciences, Volume XL-5/W4, pp. 351357.

Eastman, C., Teicholz, P., Sacks, R., Liston, K., 2008. BIM Handbook - A guide to Building Information Modeling for owners, managers, designers, engineers, and contractors. John Wiley \& Sons, Inc..

Fai, S., Graham, K., Duckworth, T., Wood, N. and Attar, R., 2011. Building Information Modeling and Heritage Documentation. CIPA 2011 Conference Proceedings: XXIIIrd International CIPA Symposium, 8 pages.

Hu, S.M., Li, Y.F., Ju, T., Zhu, X., 2001. Modifying the shape of NURBS surfaces with geometric constraint. Computer-Aided Design, 33, 903-912.

Murphy, M., McGovern, E., Pavia, S., 2013. Historic Building Information Modelling - Adding intelligence to laser and image based surveys of European classical architecture. ISPRS Journal of Photogrammetry and Remote Sensing, 76, 89-102.

Oreni, D., Brumana, R., Cuca, B., Georgopoulos, A., 2013. HBIM for conservation and management of built heritage: Towards a library of vaults and wooden bean floors. In CIPA 2013 XXV International Symposium, ISPRS Annals, volume 164, pages $1-6$.

Oreni, D., Brumana, R., Della Torre, S., Banfi, F., Barazzetti, L., Previtali, M., 2014. Survey turned into HBIM: the restoration and the work involved concerning the Basilica di Collemaggio after the earthquake (L'Aquila). ISPRS Annals of the Photogrammetry, Remote Sensing and Spatial Information Sciences, vol.II, pages 267-273.

Patraucean, V., Armeni, I., Nahangi, M., Yeung, J., Brilakis, I., Haas, C., 2015. State of research in automatic as-built modelling, Advanced Engineering Informatics, 29 (2), 162-171.

Piegl, L. A., Tiller, W., 1997. The NURBS book. Springer.

Quattrini, R., Malinverni, E.S., Clini, P., Nespeca, R., Orlietti, E., 2015. From TLS to HBIM. High quality semantically-aware $3 \mathrm{~d}$ modeling of complex architecture. The International Archives of the Photogrammetry, Remote Sensing and Spatial Information Sciences, Volume XL-5/W4, 367-374.

Tang, P., Huber, D., Akinci, B., Lipman, R., Lytle, A. (2010). Automatic reconstruction of as-built building information models from laser-scanned point clouds: A review of related techniques. Automation in Construction, 19, 829-843.

Volk, R., Stengel, J., Schultmann, F., 2014. Building Information Modeling (BIM) for existing buildings - Literature review and future needs. Automation in Construction, 38, 109-127. 